\title{
Observations of plasmons and edge magnetoplasmons in voltage-tunable dots in GaAs/AlGaAs heterostructures
}

\author{
N K Patel, T J B M Janssen, J Singleton, M Pepper, J A A J Perenboom, \\ D A Ritchie, J E F Frost and G A C Jones \\ Cavendish Laboratory, Madingley Road, Cambridge CB3 OHE, UK
}

Received 24 September 1992

\begin{abstract}
Large arrays of quantum dots have been defined in a GaAs/AlGaAs heterostructure using a Schottky gate that allows simultaneous measurement of the capacitance and the far-infrared (FIR) transmission. With zero applied gate voltage $\left(V_{g}\right)$, the FIR magnetotransmission shows the characteristic cyclotron resonance $(\mathrm{CR})$ and a 2D magnetoplasmon. By applying a negative $V_{g}$, quantum dots are defined and a new response is observed, which is described using an edge magnetoplasmon model. Furthermore, the measurements allow the dot radius to be extracted and show that the radius can be varied by up to $50 \%$ by altering $V_{\mathrm{g}}$.
\end{abstract}

Recently there has been a growing interest in the properties of quantum dot systems, produced on high-mobility heterostructures by either using metal gates [1-3], or deep mesa etching $[4,5]$. This work has followed on from the extensive studies performed on one-dimensional wires using transport $[6,7]$ and optical $[8,9]$ techniques. In the case of quantum dots, the electrical contacts necessary for transport measurements are difficult to produce, and this has meant that the majority of the experiments have concentrated on capacitance studies $[10,11]$ or optical spectroscopy [1-5]. In this paper, a structure has been designed such that both capacitance and far-infrared (FIR) transmission experiments can be performed on the same sample. The two measurements provide complementary information, which is useful for interpreting the results.

A schematic diagram of the structure is shown in figure 1(a), it consists of a standard (2DEG single heterostructure (referred to as the top 2DEG), with an additional Si $\delta$-doped conducting layer $2000 \AA$ below. This second conducting layer provides a back contact that remains continuous even when the dots become defined. The back contact provides a ground plane relative to which the gate voltage is applied; this is necessary to produce the electrostatic squeezing that defines the dots and is also required to measure the capacitance. The added advantage of using a $\delta$-doped layer is that it is transparent to FIR radiation and so transmission experiments are possible with this structure. The mesa was defined using optical lithography and wet etching, with the sample wedged to an angle of $2^{\circ}$. Electrical ohmic contacts were formed with NiAuGe annealed sufficiently to make contact to both conducting layers. The dots were defined on the mesa using electron beam lithography with high-resolution negative (HRN) resist. This process produced a large array of pillars of resist, with 

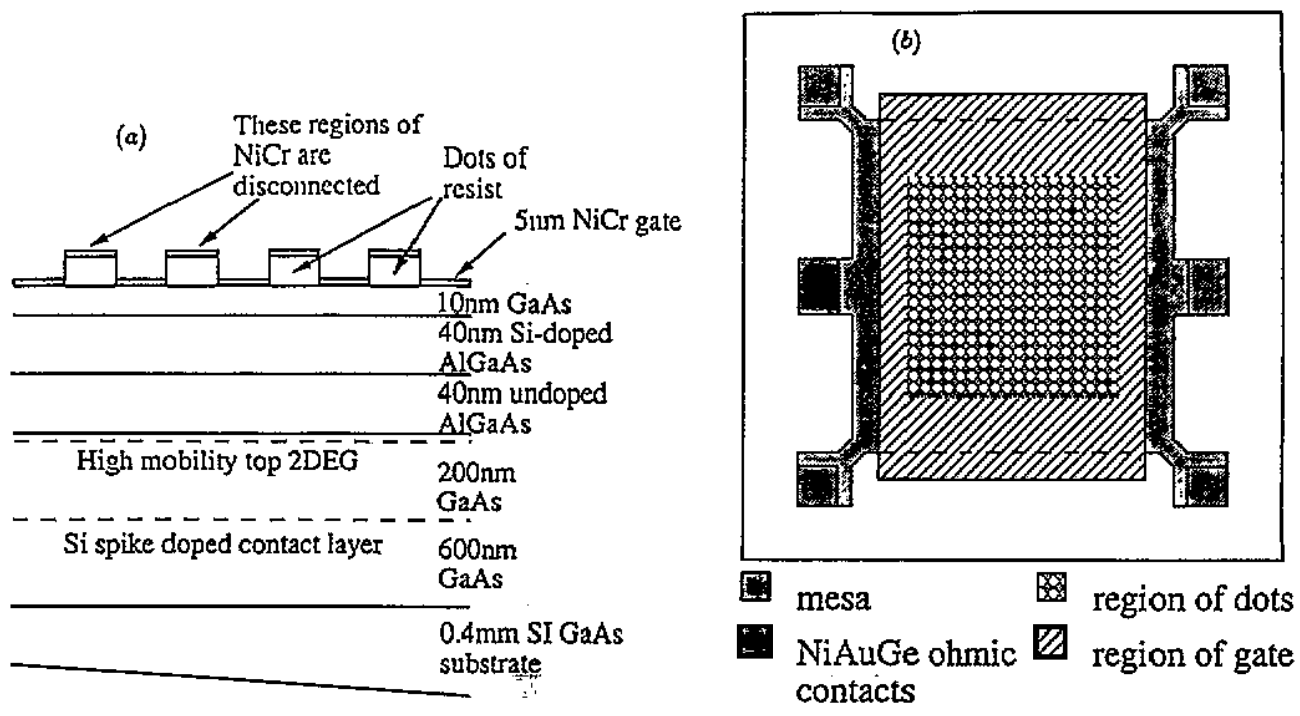

Figure 1. (a) The structure of the device with the top-high mobility $2 \mathrm{DEG}$ and the bottom low-mobility $\delta$-doped $2 \mathrm{DEG}$. (b) The final geometry of the device with the central region of dots covering an area of $2 \mathrm{~mm}^{2}$.

a periodicity of $2.5 \mu \mathrm{m}$. Each dot had a radius of $0.5 \mu \mathrm{m}$ and a resist height of $0.5 \mu \mathrm{m}$, and a total sample area of $2 \mathrm{~mm}^{2}$ was covered by the array of dots. A gate is produced by evaporating a $50 \AA$ thick layer of $\mathrm{NiCr}$ that acts as the semitransparent gate over the dots of resist. The dots are defined in the top 2DEG by applying a negative gate voltage that depletes electrons selectively from under the gated regions. The $\mathrm{NiCr}$ covering the pillars of resist is disconnected from the rest of the NiCr by the steep walls of the resist. Hence, the regions under the resist are not affected by the gate voltage and remain undepleted, and it is this region that forms the quantum dots to be investigated. The back contact, provided by the Si $\delta$-doped layer, is spatially further away from the metal gate than the top 2DEG and so remains relatively unaffected by the gate voltage. Figure $1(b)$ shows a plan view of the completed device.

The capacitance measurements are primarily used to accurately monitor the carrier density, both before and after the dots are defined. This is possible since the capacitance depends on the density of states at the Fermi energy [12]; hence, the magnetocapacitance oscillations similar to Shubnikov-de Haas oscillations give directly the carrier density which is vital for interpreting the optical spectra. The dots used are relatively large $(1 \mu \mathrm{m}$ diameter) and clear effects are seen in the system due to classical magnetoplasmons [13], in both the unbounded (before the dots are defined) and bounded (when the quantum dots are defined) cases. These plasmons are studied as a function of both the carrier density and the size of the dot by varying the gate voltage.

Characterization of the sample by transport measurements was carried out in order to extract mobilities of the two separate conducting layers and to ensure that the gate was depleting the top $2 \mathrm{DEG}$. The resistance and capacitance of the sample show a clear turn-on when the gate voltage is applied. The resistance increases and then remains virtually constant above a gate voltage of $-0.2 \mathrm{~V}$, due to the top 2DEG 
depleting and the bottom 2DEG remaining unaffected by the gate. Simultaneously, the depletion of the top 2DEG causes a decrease in the capacitance as shown in figure 2. A detailed study of the magnetoresistance was carried out and this indicated the presence of the two separate conducting layers. A large positive magnetoresistance followed by Shubnikov-de Haas oscillations was observed. Analysis similar to that of Kane et al [14] of the magnetocapacitance data results in values for the mobility of 1 $\times 10^{6}$ and $1 \times 10^{4} \mathrm{~cm}^{2} \mathrm{~V}^{-1} \mathrm{~s}^{-1}$ for the top 2DEG and lower back contact respectively, with carrier densities of $2.7 \times 10^{11}$ and $2 \times 10^{12} \mathrm{~cm}^{2}$.

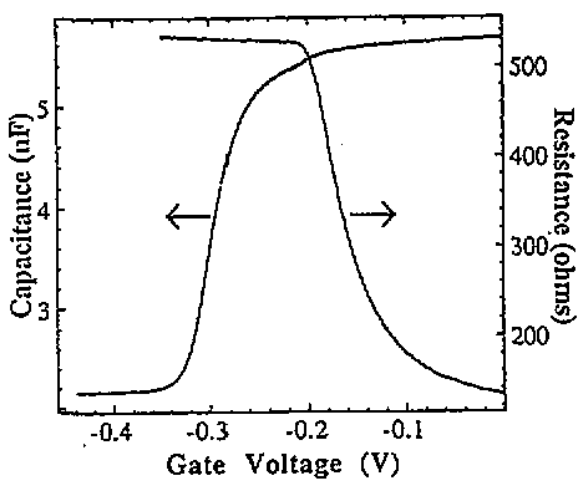

Figure 2. The resistance and capacitance of the device are shown as a function of the gate voltage. They both undergo a transition at a similar gate voltage which comresponds to the formation of the dots of electrons in the top $2 \mathrm{DEG}$.

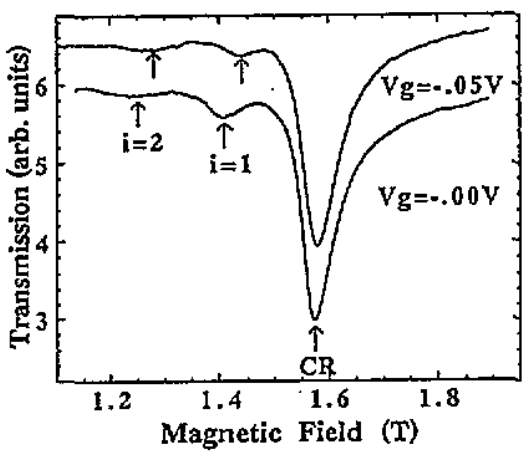

Figure 3. The magnetocapacitance spectrum for zero gate voltage (lower trace) and for $-50 \mathrm{mV}$ (upper trace) using $469 \mu \mathrm{m}$ radiation. The cyclotron resonance (CR) has a transmission dip of nearly $50 \%$ and the $2 \mathrm{D}$ plasmons also appear. The grating harmonicinduced plasmon, marked $i=2$, is also observed. As the gate voltage is applied the plasmon peaks move to higher field and the $\mathrm{CR}$ is unmoved.

The measurements were undertaken in a ${ }^{3} \mathrm{He}$ cryostat at temperatures of approximately $400 \mathrm{mK}$. Unpolarized light from an optically pumped FIR laser was coupled to the sample sitting in the cryostat via light pipes. The wavelength range 
available was $70-569 \mu \mathrm{m}$ and the transmitted signal was measured using a bolometer held at $12 \mathrm{~K}$ situated directly below the sample. A reference bolometer was also placed above the sample to measure the laser power, which was used to normalize the transmitted signal, and the cryostat was placed in a $20 \mathrm{~T}$ Bitter magnet. The capacitance measurements wee performed by applying a small oscillating voltage superimposed on the gate voltage. The resulting oscillating current was measured between the gate and the conducting layers using a lock-in amplifier, with the outof-phase signal used to measure the capacitance between the gate and the 2DEG. In the limit of a pure capacitance, the out-of-phase signal is directly proportional to the capacitance, and this is valid as long as the resistive component, measured by the inphase signal, is small. To ensure that the in-phase signal was negligible, a frequency below $10 \mathrm{kHz}$ was used.

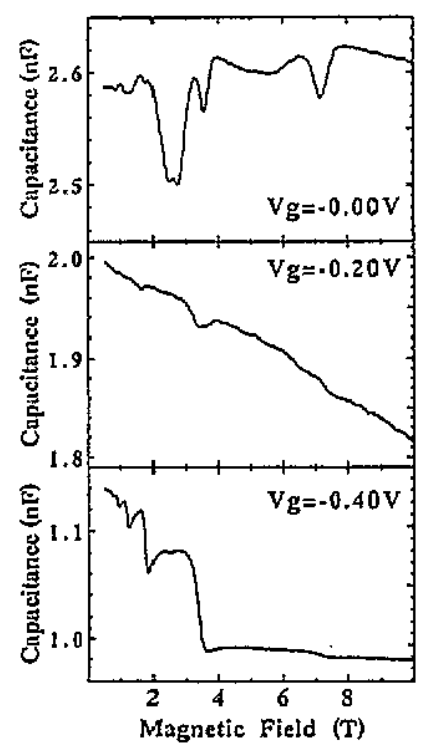

Figure 4. The magnetocapacitance is shown in the range $0-10 \mathrm{~T}$ at three different gate voltages. These correspond to the uniform $2 \mathrm{DEG}\left(V_{B}=-0.00\right)$, the highly modulated case where the oscillations nearly disappear $\left(V_{B}+-0.20\right)$ and the case when the dots become isolated $\left(V_{g}=-0.40\right)$.

The transmission spectrum taken with $469 \mu \mathrm{m}$ radiation at zero gate voltage is shown as a function of magnetic field in figure 3. The prominent feature is the cyclotron resonance (CR) peak that has a narrow linewidth and nearly $50 \%$ absorption, close to the maximum expected for unpolarized light [13]. The narrow linewidth indicates the high mobility of the sample used in the experiment and the high absorption is a result of the $\mathrm{NiCr}$ grating, which has been shown both experimentally and theoretically to produce absorption above 50\% [13,15]. Using the standard relationship for the cyclotron frequency $\omega_{c}=e B / m^{*}$ we obtain a value of $0.069 \mathrm{~m}_{\mathrm{e}}$ for the effective mass, which agrees with the value quoted for GaAs/AlGaAs heterostructures of this carrier density. Side peaks were also observed in the magnetotransmission, which were identified as $2 \mathrm{D}$ magnetoplasmons with a 


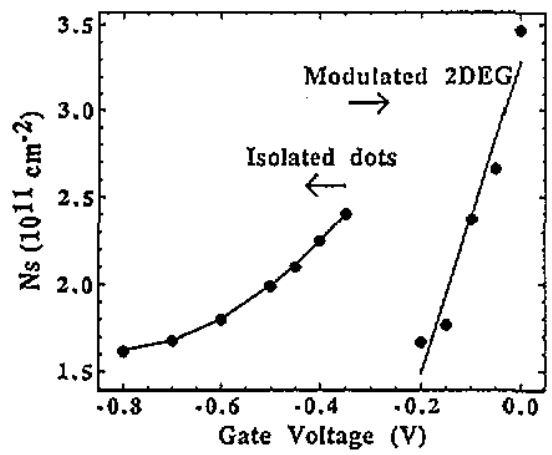

Figure 5. The electron carrier density is extracted from the capacitance data. A clear transition occurs above $-0.20 \mathrm{~V}$ at which point we move from measuring the density in the regions surrounding the dots to measuring the signal solely from the dots.

frequency $\omega_{\mathrm{p}}$ given by

$$
\omega_{\mathrm{p}}(B)^{2}=\omega_{\mathrm{p}}(B=0)^{2}+\omega_{\mathrm{c}}^{2} .
$$

These side peaks were observed due to the array of dots acting as a $2 \mathrm{D}$ grating resulting in the coupling of the radiation to plasmons with wavevectors given by

$$
q=2 \pi i / a
$$

where $a$ is the array period and $i$ is an integer. The plasmon energy in zero magnetic field can now be calculated using a simple classical model [13]

$$
\omega_{\mathrm{p}}(B=0)^{2}=N_{\mathrm{s}} e^{2} i q / 2 m^{*} \epsilon_{0} \epsilon_{\mathrm{eff}}
$$

where $N_{\mathrm{s}}$ is the carrier density and $\epsilon_{\text {eff }}$ is the effective dielectric constant. For the dielectric constant we need to take into account the screening of the gate; assuming the gate acts as a perfect grounding plate the effective dielectric constant is given by the following formula $[13,16]$

$$
\epsilon_{\mathrm{eff}}=\left[\epsilon_{1}+\epsilon_{2} \operatorname{coth}(q d)\right] / 2
$$

where $\epsilon_{1}$ is the dielectric constant for GaAs, $\epsilon_{2}$ is the dielectric constant for AlGaAs and $d=900 \AA$ is the depth of the 2DEG from the gate metal. Using the above relationships, taking $i=1$ and using the value of the carrier density determined from the capacitance data, experimental and theoretical values of $\omega_{p}$ within $10 \%$ of each other were obtained. This is very reasonable agreement, since no variable fitting parameters are used. The second peak at lower magnetic field corresponds to the $i=2$ value for the wavevector. This type of harmonic has been observed in experiments using conventional 1D grating couplers [13]. The experiments were repeated using different FIR wavelengths and showed the same features with the magnetoplasmons having the strongest oscillator strengths when illuminated with long-wavelength radiation. The energies of the plasmons obtained for the different wavelength illumination all agreed to within experimental accuracy. A further confirmation of the identity of the 2D plasmons was obtained by flashing an LED whilst 


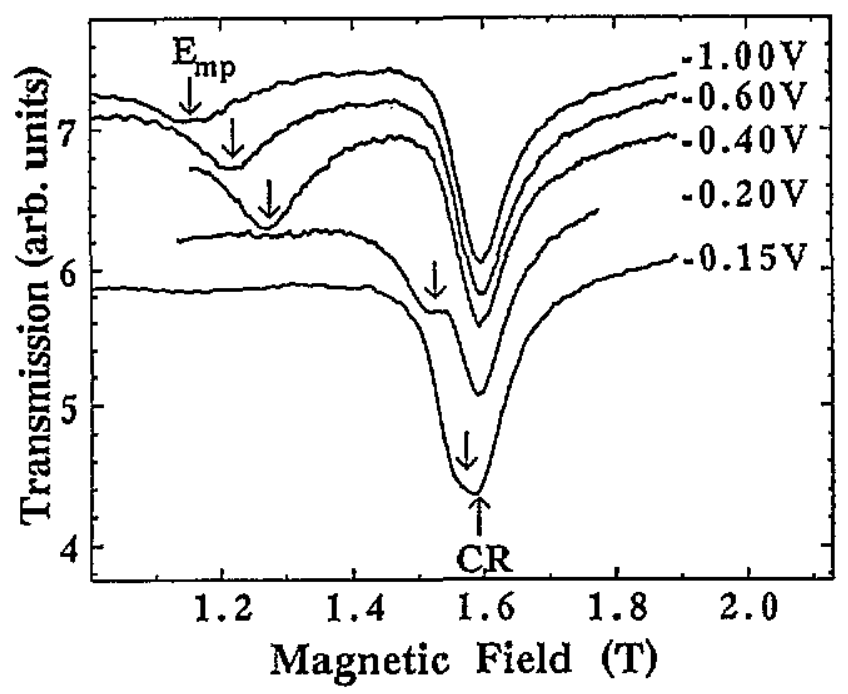

Figure 6. The magnetotransmission taken with $469 \mu \mathrm{m}$ radiation is shown for different gate voltages. The cyclotron resonance in unaffected and the magnetoplasmon $\left(E_{\mathrm{mp}}\right)$ moves to lower field. This corresponds to an increase in the plasmon energy related to the decrease in the dot size with gate voltages.

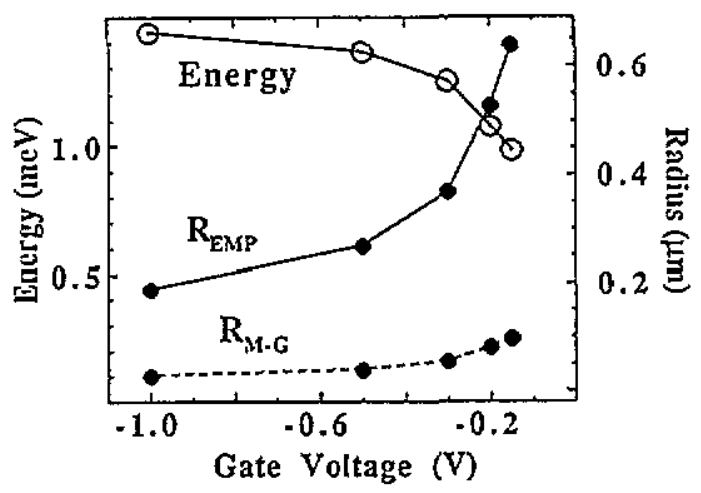

Figure 7. The open circles show the energy dependence of the plasmon bounded inside the $2 \mathrm{DEG}$ with gate voltage (calculated for zero magnetic field). The closed circles show the calculated radius for different gate voltages. The edge magnetoplasmon model and the Maxwell-Garnet depolarization model are used to obtain the dot radius $R_{\text {EMP }}$ (full curve) and $R_{\mathrm{M}-\mathrm{G}}$ (broken curve) respectively.

the sample was cold and repeating the measurements. The LED induces carriers by the persistent photoconductive effect and enhances the mobility; the higher carrier concentration resulted in an increase in the percentage absorption and the higher mobility produced a narrowing of the cyclotron resonance peak. The increase in the carrier density also caused the $2 \mathrm{D}$ magnetoplasmon peaks to move to lower field which corresponds to a higher energy as expected from equation (3).

Increasing the negative gate voltage had the effect of reducing the carrier density, as obtained from analysing period of the oscillations that appear in the magnetocapacitance. Correspondingly, the energy of the plasmons was reduced 
according to equation (3). This is seen in figure 3 where for a gate voltage of $-0.05 \mathrm{~V}$ both magnetoplasmons peaks have moved closer to the cyclotron resonance peak. This continues until all traces of the magnetoplasmons disappear. At a gate voltage of $-0.20 \mathrm{~V}$ the magnetocapacitance also becomes unclear with the oscillations disappearing. As the negative gate voltage is further increased, the magnetocapacitance oscillations re-emerge. This transition is clearly seen in the magnetocapacitance traces in figure 4 and occurs when the 2DEG surrounding the dots becoming completely depleted; the capacitance is now due solely to the electrons in the dots. The oscillations in the magnetocapacitance are used to obtain the electron carrier density, shown in figure 5 , which has a discontinuous change that occur when the dots are formed. As the dots are formed in the top 2DEG a new side peak appears in the FIR transmission that must be related to the dots. This peak moves to lower field with increasing gate voltage, as shown in figure 6 , which is the opposite to the magnetoplasmon which moved to higher field with increasing gate voltage. The energy of this peak obeys the following relationship in a magnetic field, as shown by Liu et al [17]

$$
\omega \pm+ \pm \omega_{\mathrm{c}} / 2+\left[\left(\omega_{\mathrm{c}} / 2\right)^{2}+\omega_{0}^{2}\right]^{1 / 2}
$$

where $\omega_{0}$ is the energy of the observed plasmon in zero magnetic field. In our measurements we only see the positive branch, whereas Fourier transform interferometer measurements have also detected the lower negative branch [2-4]. The magnetic field dependence of the negative branch is small and results in a peak in the magnetotransmission spectrum too broad to measure. For all gate voltages the cyclotron resonance peak also remains in the spectrum, but much diminished in magnitude; this does not completely disappear due to the presence of ungated regions on the sample. We now concentrate on the new peak which appears concurrently with the formation of the dots and is modelled using two different approaches. The first method uses a classical Maxwell-Garnet theory of depolarization, as used by Allen $e t$ al [4], which gives the energy as

$$
\omega_{0}^{2}=N_{\mathrm{s}} e^{2} / 16 m^{*} \epsilon_{0} \epsilon_{\mathrm{eff}} R
$$

where $R$ is the radius of the dot, $\epsilon_{\mathrm{eff}}$ is the same as in equation (4) and $N_{\mathrm{s}}$ is the carrier density obtained from the capacitance. Figure 7 shows the values of $R$ extracted using this model. When the dots are first formed we expect a radius of $0.5 \mu \mathrm{m}$, as measured in SEM photographs of the sample. This corresponds to an energy of only $0.45 \mathrm{meV}$ using the above model, which is lower than the measured value if we assume the dots are first formed at $-0.30 \mathrm{~V}$. However, a better agreement is found if we use the edge magnetoplasmon model $[16,18,19]$ for a bounded plasmon in a 2DEG. Apart from the numerical constant this is equivalent to the 2D magnetoplasmon energy given by equation (3) with the wavevector $q=2 \pi / 2 \pi R$ determined by the circumference of the dot of radius $R$

$$
\omega_{0}^{2}=\left(\frac{2}{3}\right)^{1 / 2} N_{\mathrm{s}} e^{2} / 2 m^{*} \epsilon_{\mathrm{v}} \epsilon_{\mathrm{eff}} R .
$$

This gives an energy of $1.15 \mathrm{meV}$ for a $0.5 \mu \mathrm{m}$ dot radius, which is close to the value we obtain experimentally. This indicates that the edge magnetoplasmon model is better suited to describe our data than the classical Maxwell-Garnet theory. Using 
this model further we see in figure 7 that the radius of the dots is reduced by nearly $50 \%$ due to the application of the gate voltage.

In summary, our experiments show that simultaneous measurements of capacitance and FIR transmission is possible in quantum dots. We have observed 2D magnetoplasmons, due to the 2D array of dots acting as a grating coupler, which fit accurately with theoretical predictions. The energies of both plasmons scale as expected with magnetic field and carrier density. Once the dots are defined an absorption peak is seen which we can describe by an edge magnetoplasmon model, and using this we see that the dot radius can be reduced by up to $50 \%$ by varying the gate voltage.

\section{References}

[1] Sikorski Ch and Merkt U 1989 Phys. Rev. Lett. 622164

[2] Alsmeier J, Batke E and Kotthaus I P 1990 Phys. Rev. B 411699

[3] Lorke A and Kotthaus J P Phys. Rev: Lett. 642559

[4] Allen S J Jr, Stormer H L and Hwang J C M 1983 Phys. Rev. B 284875

[5] Demel T, Heitmann D, Grambow P and Ploog K 1990 Phys. Rev. Lett. 64788

[6] Thornton T J, Pepper M, Ahmed H, Andrews D and Davies G J 1986 Phys. Rev. Lett. 561198

[7] Demel T, Heitmann D, Grambow P and Ploog K 1988 Appl. P/yss. Lett. 532176

[8] Alsmeier J, Batke E and Kotthaus J P 1989 Phys. Rev. B 4012574

[9] Brinkop F, Hansen W, Kotthaus J P and Ploog K 1988 Phys. Rev B 376547

[10] Hansen W, Smith T P III, Lee K Y, Brum J A, Knoedler C M, Hong J M and Kern D P 1989 Phys. Rev. Lett. 622168

[11] Ismail K, Smith T P III, Masselink W T and Smith H I 1989 Appl Phys. Lest. 552766

[12] Smith T P III, Goldberg B B, Stiles P J and Heiblum M 1985 Phys. Rev. B 322696

[13] Batke E, Heitmann D and Tu C W 1986 Phys. Rev: B 346951

[14] Kane M J, Apsley N, Anderson D A, Taylor L L and Kerr T 1985 J. Phys. C: Solid State Phys. 18 5629

[15] Zheng L, Schaich W L and MacDonald A H 1990 Phys. Rev. B 418493

[16] Que W-M and Kirczenow G 1989 Phys. Rev. B 395998

[17] Liu C T, Nakamura K and Tsui D C 1989 Apph Phys. Leth 55168

[18] Wu J-W, Hawrylak P and Quinn J J 1985 Phys. Rev. Lett. 55879

[19] Fetter A 1985 Phys. Rev. B 327676 